\title{
La calidad de los medios y el uso de fuentes perio- dísticas en la prensa local de referencia en España ${ }^{1}$
}

\author{
Ana RodRÍGUEZ REY \\ Universidad de Sevilla \\ anarodrey@gmail.com \\ Alicia Enguix GonZÁleZ \\ Universidad de Sevilla \\ aenguix@us.es \\ José Luis ROJAS TORRIJOS \\ Universidad de Sevilla \\ jlrojas@us.es \\ María del Mar GARCíA GORDILLO \\ Universidad de Sevilla \\ marggordillo@us.es
}

Recibido: 5 de diciembre de 2014

Aceptado: 8 de mayo de 2015

\begin{abstract}
Resumen
La evaluación de las fuentes informativas es esencial para medir la calidad del trabajo periodístico. La peculiaridad de la prensa local, que se ocupa de las noticias más próximas, las que atañen a las necesidades cotidianas de la audiencia, se convierte en el banco de pruebas idóneo para analizar pormenorizadamente cómo inciden el número, características, procedencia, e iniciativa de las fuentes en la calidad periodística. Este artículo presenta los resultados de un estudio cuantitativo-cualitativo sobre las noticias publicadas en la edición impresa de diarios locales españoles de referencia, con el fin de analizar la calidad de las informaciones con mediciones concretas, según el uso de las fuentes por parte de los redactores y a partir de la aplicación del método del Valor Agregado Periodístico (VAP) y de técnicas estadísticas.
\end{abstract}

Palabras clave: calidad periodística, periodismo de proximidad, fuentes de información, prensa local.

\section{The quality of media and the use of sources in the local reference press in Spain}

\begin{abstract}
The evaluation of sources of information is essential to measure the quality of journalistic work. The special nature of local press, that deal with the closest news and satisfy the everyday needs of the audience, becomes the most valid sample to know the real incidence the sources of information have in the quality in journalism according to their number, typology, origin and own initiative. This article shows the results of a qualitative and quantitative study on the news published in the print edition of Spanish local reference newspapers in order to analyse them by qualitatively measuring of the informative product according to the way reporters use their sources and from the application of Added Value in Journalism method (VAP) and statistical techniques.
\end{abstract}

Keywords: quality journalism, journalism of proximity, sources, local press.

1 Este trabajo ha sido realizado por el Grupo de Investigación Estudio de Medios para un Periodismo de Calidad (SEJ-001) y junto con otro incluido en esta misma publicación "El uso de fuentes periodísticas en las secciones de Local, Economía y Cultura en el periodismo de proximidad" dan una imagen de conjunto del uso que los medios locales hacen de las fuentes informativas desde un doble punto de vista: global como periodismo de proximidad y según temáticas y secciones. 


\section{Referencia normalizada}

RODRÍGUEZ REY, Ana; ENGUIX GONZÁLEZ, Alicia; ROJAS TORRIJOS, José Luis; y GARCÍA GORDILLO, María del Mar (2015): "La calidad de los medios y el uso de fuentes periodísticas en la prensa local de referencia en España". Estudios sobre el Mensaje Periodístico. Especial noviembre "Periodismo e información de calidad", págs.: 85-100. Madrid, Servicio de Publicaciones de la Universidad Complutense.

Sumario: 1. Introducción. 2. Metodología; 2.1. Muestra. 3. Resultados; 3.1. Si el periodista acude al lugar en que se produce la noticia; 3.2. Si es el periodista quien toma la iniciativa, según sea el tema de la noticia institucional o no institucional; 3.3. Si el periodista es quien toma la iniciativa independientemente de la temática; 3.4. Si accede a las fuentes necesarias en noticias que plantean intereses contrapuestos; 3.5. Si el número de fuentes suele estar en relación con la calidad de la noticia; 3.6. Número de fuentes según la temática institucional o no institucional; 3.7. Número de fuentes y presencia del periodista; 3.8. Muchos acontecimientos no se entienden si no se conocen los antecedentes; 3.9. Las consecuencias que se derivan de los acontecimientos deben ser también conocidas por la audiencia; 3.10. La identificación de las fuentes es otro de los parámetros de calidad; 3.10.1. Desde el punto de vista de las fuentes; 3.10.2. Desde el punto de vista de las propias noticias. 3.11. Relación entre noticias institucionales y fuentes institucionales. 4. Conclusiones. 5. Referencias bibliográficas.

\section{Introducción}

Las fuentes no son un recurso importante en el periodismo, son la sustancia del periodismo. Desde el momento en que la labor periodística consiste en "contar a la gente lo que le pasa a la gente" (Lippmann 1965: 280), "poner en conocimiento del ciudadano la información que precisa para tener criterio y actuar con elementos de juicio" (Kovach y Rosenstiel, 2003: 24) se pone en evidencia que el profesional de la información tendrá que contactar con los protagonistas o los testigos de los hechos que merecen contarse; hallar confidentes dispuestos a poner al descubierto lo que intereses inconfesables o ilícitos pretenden ocultar; conseguir interlocutores, documentos, expertos capaces de proporcionar la información completa sobre hechos relevantes que el ciudadano necesita saber (Bezunartea et al., 1998: 78; Fishman, 1980: 107). Las relaciones con las fuentes proporcionan a los periodistas una de las pocas facetas en la autonomía de su trabajo: las fuentes constituyen su patrimonio personal (Bezunartea 1988: 167).

Las fuentes son recursos imprescindibles para construir el relato y contar la noticia y para conferir credibilidad tanto a la información como al propio procedimiento usado para elaborarla y emitirla (Diezhandino, 2007: 63).

En el periodismo local, los reporteros, los protagonistas de las noticias y los receptores forman un triángulo que se mueve en los mismos espacios. Las noticias locales tratan, o deberían tratar, cuestiones que atañen a la vida cotidiana. Si el medio responde al ritmo vital de la sociedad a la que se dirige debe reflejar las interacciones y ser tan sensible a los promotores de los temas que son objeto de noticia, como a los que se sienten concernidos por los propios temas.

El compromiso del informador con el rigor y la precisión de las noticias son mucho más altos en el periodismo de proximidad que cuando los hechos ocurren en lugares alejados. En el medio local, los receptores identifican los escenarios y a los protagonistas; a veces han podido ser testigos de los acontecimientos. En un amplio estudio 
del Media Research Center Commisioned by Media Trust realizado en Gran Bretaña ${ }^{2}$ sobre cómo el periodismo local debe responder a las necesidades cívicas, democráticas y comunitarias clasificaron "las genuinas necesidades de la calidad de las noticias y el periodismo". El resultado de las entrevistas puso de manifiesto que los receptores detectaban falta de información sobre lo que ocurría a su alrededor, escasez de periodismo de investigación, pedían que se notara que el periodista conoce a fondo las noticias, que profundizara en ellas: "quieren profundidad analítica, escepticismo con respecto a los que ocupan el poder, contexto significativo que permita debates profundos". Entienden que la buena información local es también la que da voz a los sin voz; que escucha y representa a la gente corriente. Que actúa como cuarto poder, lo que significa que no solo debe revelar la actividad de las distintas administraciones públicas y las entidades privadas relevantes, sino las motivaciones que hay tras ellas.

En el estudio What the public expects of local news (Heider et al., 2005: 952), sus autores, a través de entrevistas y cuestionarios, descubrieron que a los consumidores de medios locales lo que les interesa primero es el rigor (94\%), después la información equilibrada, no manipulada (84\%); que incluya distintos puntos de vista (69\%); que entienda y cuide la comunidad (ambos el 68\%); que ofrezca soluciones a los problemas (51\%); cuestiones como proporcionar temas para debate o ejercer la función de watchdog u ofrecer la información con rapidez no llegaron al apoyo de más del $50 \%$ de los consultados. La misma encuesta entre periodistas, da respuestas con matices muy distintos a la cuestión de cuáles son las características más importantes de las noticias; si bien el rigor y la falta de prejuicios ocupan los primeros lugares entre los profesionales, para estos el papel de watchdog es de lo más relevante (70\%), mientras que dar rápidamente la información tiene una brecha del 59\% al 35\% entre periodistas y consumidores de noticias. De estas diferencias de percepción deducían los investigadores que difícilmente iba a haber buena sintonía entre el contenido de los medios locales y el público (Heider et al., 2005: 259).

Las preguntas clave sobre cuáles son las características centrales del buen periodismo local, qué papel deben cumplir las noticias en la vida de la comunidad local, qué necesita conocer el público, remiten a plantear a qué fuentes debe recurrir el periodista para responderlas satisfactoriamente y cuál debe ser su método de trabajo.

Si las "fuentes hacen las noticias", como dijo Sigal (1986: 93) no solo serán quienes protagonizan la noticia, sino que serán también quienes darán la versión de lo que ocurre. Que los periodistas que deben cubrir los grandes centros de decisión política o económica tengan que limitarse a un relativamente pequeño círculo de fuentes, es mucho menos explicable en el ámbito local. Sin embargo, como ya observaba en 1964 Walter Gieber en un estudio sobre noticias locales:

"Los periodistas, a pesar de que se crean agentes actuando libremente, buscando e investigando sus propias historias, raramente son independientes de sus fuentes, al contrario

2 "Meeting the news needs of local communities", News Research by Goldsmiths Leverulm Media research Center Commisioned by Media Trust, http:/www.mediatrust.org/uploads/ [consultado el 1/10/2013]. 
generan noticias basadas en la información de fuentes que se benefician más de ello que los propios periodistas" (Gieber, 1964: 291).

Y no sería problema si tal fuera de interés público y, sobre todo, si contaran además con otras fuentes para verificar y completar sus noticias. La noticia de una sola fuente, no logra llegar al meollo de la cuestión o no alcanza a completar la historia, sino que pasan por alto puntos de vista alternativos y perspectivas relevantes para los lectores (O’Neill, O'Connor 2008: 492).

Que los periodistas locales acudan regularmente a los centros de abundante producción de noticias: gobiernos autonómicos ayuntamientos, parlamentos, policía, tribunales, es una obligación puesto que generan acontecimientos de interés general para los ciudadanos. Pero entre todos, fuentes oficiales convocantes y periodistas, acaban formando un círculo cerrado que ignora a los receptores como entes activos, como ciudadanos que desearían actuar como interlocutores de los distintos poderes y, sobre todo, tener voz ante los medios.

La literatura académica sobre el ejercicio del periodismo ha denunciado desde hace décadas la servidumbre de los periodistas respecto de la iniciativa de fuentes interesadas y su falta de autonomía para decidir los temas y los enfoques de las noticias que precisa el ciudadano. A los estudios que ya se han convertido en clásicos como los Gaye Tuchmann (1978) y Leon V. Sigal (1973) y Herbert Strenz (1989), Herbert Gans (1980) el ya mencionado Water Giebert se han añadido en épocas más recientes los que establecen el nexo entre la dependencia de las fuentes y el establecimiento de la agenda, cuestión que se considera como el más poderoso efecto de los medios, como hace notar Soledad Gallego Díaz (2013) en un debate sobre periodismo de investigación.

La queja es que los periodistas se enfrentan a un vasto número de paraperiodistas como los ha bautizado Schudson (2003:3), gabinetes de comunicación, empresas de relaciones públicas, portavoces políticos, instituciones públicas, corporaciones u ONG que apenas dejan tiempo disponible a los periodistas para hablar con sus contactos, cuidar fuentes... seguir temas propios se ha convertido en un lujo. Así que como se expone en The Passive Journalism, los periódicos locales están dejando de cumplir su viejo papel de guardianes de la verdad y de notarios de la actualidad:

“La prensa local necesita despertar rápido y reinventarse a sí misma antes de que los únicos lectores que queden sean los funcionarios, los relaciones públicas y grupos de presión que han arrastrado a los editores y reporteros sin imaginación" (O’Neill, O'Connor, 2008: 498).

Cuando se define periodismo de calidad se habla de la exigencia de veracidad, de comprobación, de contexto, de pluralismo, de interés público, todos ellos rasgos esenciales, pero que precisan un desarrollo más concreto de modo que su puesta en práctica satisfaga las necesidades de las audiencias a las que se dirige. Las señas de identidad del buen periodismo se encuentran en su capacidad de contextualizar las informaciones, aportar datos, generales y particulares comparativos y evolutivos, cuanto dé sentido y significado y permita la comprensión del tema y lo aproxime al lector como ciudadano. Y todo ello está relacionado con las fuentes. 
La transparencia es otra de las condiciones de la calidad del periodismo que remite al tratamiento de las fuentes. El modo más directo de respetar la inteligencia del receptor es revelarle las fuentes y el método de obtención de datos: así, cuando la información procede de una fuente interesada, identificarla permitirá al lector saber que se encuentra ante una versión sesgada. Del mismo modo que el lector debe saber que la iniciativa de una noticia la ha tenido la fuente interesada y no el periodista. Tampoco es intrascendente el lugar físico donde se ha producido el encuentro. Pilar Diezhandino (2007: 69) señala la frecuente ausencia de un dato fundamental para interpretar la noticia, como es el dónde se ubica físicamente su origen. El problema que denuncia Diezhandido también ha llamado la atención en EEUU donde un estudio de Proyect for Excelence in Journalism realizado en la campaña presidencial de 2004 venía a demostrar que más de cuatro de cada diez declaraciones aparecían en los medios sin los datos sobre dónde se habían producido. Y Casero y López en "La gestión de la fuentes informativas" concluyen que:

"La gestión de fuentes se configura como un parámetro relevante de la calidad periodística. En este sentido, resulta posible establecer un conjunto de indicadores que ofrecen patrones para evaluar la excelencia a partir del uso de fuentes en las noticias. Estos se dividen en internos, cuando están vinculados directamente al proceso de producción informativa, y externos, cuando tienen que ver con los efectos sociales de la información. Entre los primeros se encuentran el estatus profesional, la verificación, la transparencia, la relevancia y la reducción de incertidumbre. Por su parte, los segundos afectan a la credibilidad, la influencia, el pluralismo y la participación" (Casero y López, 2013: 87).

Estudiar las fuentes equivale a someter a evaluación un aspecto sustancial del trabajo periodístico. Los datos cuantitativos (número de fuentes por noticia) ya son muy significativos, pero también lo son los que evidencian que el periodista se ha sentido satisfecho con la fuente oficial, institucional, "poco plural y unívoca" (Fontcuberta 1999: 64) y no ha desarrollado la noticia desde otras perspectivas, con otras fuentes; que en los temas que plantean intereses contrapuestos no se recojan los distintos implicados, a ser posible simultáneamente (mucho más si se trata de perjudicados o beneficiados); saber cuánto se ha recurrido a documentos o se ha contado con expertos; en qué proporción la agenda la han dominado las fuentes que han tomado la iniciativa; si en los temas de iniciativa propia predominan los de una sola fuente, lo que viene a significar información irrelevante, intrascendente, si acaso curiosa, en todo caso carente de profundidad.

Todas ellas cuestiones que inciden en la calidad del producto informativo que necesita el ciudadano, tanto más cuando los temas afectan directamente en su vida y sus protagonistas conviven con él. Cuando el periodista se deja atrapar por el círculo de productores interesados de noticias se olvida de que contar lo que pasa obliga a salir de ese círculo e interactuar con el ciudadano y descubrir otra actualidad no menos trascendental. 


\section{Metodología}

El VAP ${ }^{3}$ se ha revelado como un verdadero hallazgo para evaluar de un modo objetivo la calidad del trabajo periodístico (García Gordillo et al., 2013: 40). Partiendo de la base de que las fuentes juegan un papel fundamental en la labor periodística, el VAP permite un estudio de las mismas desde una doble perspectiva: el gatekeeping y el newsmaking. Sin embargo, cuando se distorsiona la labor de las fuentes al copar un papel excesivamente destacado en el gatekeeping, la herramienta puede presentar carencias.

Proponemos una ficha de análisis que abunda, algo más, en el uso que hacen de las fuentes los medios de comunicación locales, que a fin de cuentas son aquellos que tienen un acceso más directo a las mismas para la elaboración de sus textos periodísticos.

La ficha de análisis se estructura en cuatro grandes grupos de indicadores con los que se abarca el ámbito de acción del presente estudio. El primer bloque de indicadores son aquellos que definen la localización de la unidad de análisis dentro de la muestra, considerando: diario, fecha de publicación y sección. El segundo bloque corresponden a los indicadores descriptivos de la noticia analizada: firma, continuidad de la noticia, presencia en el lugar de los hechos, tipo de titulares y temas. En tercer lugar contamos con indicadores que pretenden describir las características interpretativas de los textos atendiendo a: interés de la noticia, presencia de antecedentes y de consecuencias. Para el estudio de las fuentes se ha destinado un bloque completo en el que se constata: el número de fuentes, el tipo de fuentes, la relación de la fuente con el periodista, así como de la fuente con el acontecimiento, y la atribución.

\subsection{Muestra}

Para la selección de los diarios, en su edición impresa, que se analizarán, se han considerado los siguientes parámetros:

1. El trabajo de campo se desarrolla en prensa local

2. Los periódicos elegidos son los diarios locales de referencia en su ámbito de distribución y los de mayor tirada.

3. Se han excluido los diarios nacionales, aunque incluyan información local ya que en la configuración empresarial es solo complementaria.

4. Es en la prensa local en la que la mayor proporción de contenidos es realizada de primera mano.

5. Los redactores de información local mantienen contacto directo con las fuentes.

Siguiendo los parámetros anteriores, los diarios seleccionados son:

- El Correo: http://www.elcorreo.com/

- La Voz de Galicia: http://www.lavozdegalicia.es/

- Norte de Castilla: http://www.elnortedecastilla.es/

- Ideal de Granada: http://www.ideal.es/

- Diario de Sevilla: http://www.diariodesevilla.es/

- Hoy (Badajoz): http://www.hoy.es/local/badajoz/

- Heraldo de Aragón: http://www.heraldo.es/

- Las Provincias (Valencia): http://www.lasprovincias.es/

${ }^{3}$ Para más información sobre el VAP revisar toda la bibliografía del Equipo de Investigación de la Universidad Católica de Chile: Alessandri (2001), Pellegrini (2011), Téramo (2006)... 
Por otra parte, teniendo en cuenta los objetivos del trabajo solo se analizarán las secciones de: Local, Economía y Cultura. De esta forma, serán objeto de análisis todas las páginas de cada una de estas secciones, pero seleccionando de cada página la noticia principal. En el caso de las secciones de Economía y Cultura, solo se analizarán las noticias principales de cada página que tengan carácter local.

En cuanto a la selección temporal se han elegido seis días consecutivos, del 23 al 28 de abril, en lugar de la semana construida con el fin de detectar el seguimiento de las noticias.

\section{Resultados}

El trabajo de campo desarrollado ha consistido en el análisis de 501 textos noticiosos en los ocho diarios seleccionados. Se han leído entre 36 y 80 noticias en cada diario, siendo Las Provincias y Norte de Castilla los que incluyen mayor cantidad de noticias locales (la suma de la secciones Local, Cultura y Economía), con 80, y Hoy (36) y La Voz de Galicia (48) los que lo hacen con una frecuencia menor.

\subsection{Si el periodista acude al lugar en que se produce la noticia}

Dentro del estudio hemos evaluado, en primer término, el grado de presencia del periodista en el lugar de los hechos informativos por iniciativa propia (periodismo de calle) en confrontación con el periodismo de mesa, esto es, el que se elabora desde la redacción del medio, y el de convocatorias (en este caso, la asistencia es a un acto que ha sido previamente convocado por una institución). Pues bien, en términos generales, el porcentaje de redactores que asiste a donde se produce la noticia $(35,7 \%)$ es similar al que acude a convocatorias $(30,9 \%)$ o hace periodismo de mesa $(32,3 \%)$. En el $1 \%$ de las noticias restantes, los relatos son imprecisos respecto a la presencia del periodista.

Al distinguir por diarios, observamos que Heraldo de Aragón (50,9\%) y Hoy (50\%) son los que realizan más periodismo de calle, frente a El Correo o Diario de Sevilla, que apuestan más por el periodismo de mesa, con un $43,1 \%$ y un $41,7 \%$, respectivamente. Por su parte, La Voz de Galicia, con un 37,5\% del total, es el único de los medios analizados donde predominan las informaciones elaboradas a partir de convocatorias, mientras que Norte de Castilla arroja un 38,8\% tanto en periodismo de calle como en convocatorias.

Si eliminamos del estudio aquellas noticias en las que no queda clara cuál es la presencia del periodista (relato impreciso), se puede afirmar que la aparición del periodista en el relato difiere según el diario (test chi-cuadrado ${ }^{4}$ con $\mathrm{p}$-valor $=0.0071$ ).

${ }^{4}$ Un test estadístico es una prueba que pretende estudiar la veracidad de una hipótesis planteada. Para ello, es necesario fijar previamente el nivel de significación a utilizar que es, en el caso de que la hipótesis fuera cierta (hecho que se desconoce), la probabilidad de dicha hipótesis sea rechazada. Es decir, si la hipótesis que se quiere estudiar fuera verdadera, daría la probabilidad de equivocarse. Este valor debe ser pequeño y se suele fijar como 0.05. Para obtener conclusiones de los tests estadísticos se suele calcular el llamado p-valor que proporciona el nivel de significación mínimo necesario para rechazar la hipótesis. Así, si el pvalor es mayor que el nivel de significación prefijado, no se rechazará la hipótesis (por falta 


\subsection{Si es el periodista quien toma la iniciativa, según sea el tema de la noticia institucional o no institucional}

A continuación, hemos tratado de determinar el origen de la iniciativa de ir a buscar la información y su relación con la temática de los textos publicados. De las 501 noticias analizadas inicialmente, hemos eliminado aquellas que no pueden ser atribuidas de una forma clara y en las que, por tanto, no puede deducirse el origen de la iniciativa $^{5}$. Así, para este estudio, hemos trabajado con 403 informaciones. De ellas, el $46,4 \%$ tratan de un asunto institucional público frente a un 53,6\% que versa sobre otro tipo de temática.

En relación a la iniciativa, se observan claras diferencias entre los dos tipos de temática (test exacto de Fisher con $p$-valor $=0.002$ ). En las noticias de tema institucional público la iniciativa de la fuente está presente en un $48,7 \%$ de los casos, un porcentaje bastante más elevado que en aquellas que tratan sobre otros temas (33,3\%). Según estos resultados, el trabajo de gabinetes de comunicación se hace notar más en el campo institucional público que en el privado, donde predomina la iniciativa del periodista, presente en dos terceras partes de los textos.

El estudio de la relación entre tema e iniciativa en los distintos diarios analizados arroja diferencias significativas (test chi-cuadrado con p-valor muy próximo a 0 ). Como datos más destacados a este respecto, en La Voz de Galicia, el $40 \%$ de las noticias son de tema institucional con iniciativa de la fuente, frente al $10.6 \%$ en el $\mathrm{He}$ raldo de Aragón; en Ideal el 32,1\% de las noticias son de tema no institucional con iniciativa de la fuente, frente al $12,5 \%$ en el Diario de Sevilla; en el Norte de Castilla, el 8,6\% de las noticias son de tema institucional con iniciativa del periodista, frente al $46.8 \%$ en el Heraldo; y en el Norte de Castilla el 55,7\% de las informaciones son de tema no institucional con iniciativa del periodista, frente al 23,2\% en Ideal.

Asimismo, hemos estudiado si la iniciativa depende de la temática de la noticia en cada uno de los diarios. Entre los resultados recogidos, Ideal aparece como el único diario donde predomina la iniciativa de la fuente cuando el tema no es institucional público, mientras que en Heraldo, El Correo y Hoy prevalece la iniciativa del periodista entre sus noticias institucionales, con más del $60 \%$ de los casos, frente al resto de los diarios en los que es superior la iniciativa de las fuentes (con valores entre el $25 \%$ y el $50 \%)$.

de evidencias de lo contrario); mientras que si el p-valor es menor o igual que el nivel de significación prefijado, se rechazará la hipótesis planteada, por ser evidentemente falsa.

En este trabajo se ha hecho uso de los tests chi-cuadrado de independencia y homogeneidad. El primero estudia si un carácter o variable influye en otro. El segundo estudia si la distribución de una variable es idéntica para las diferentes condiciones que marca la segunda variable. Ambos tests son equivalentes. Se ha aplicado la corrección de Yates del test chi-cuadrado cuando ha sido necesario. Cuando las dos variables tomaban solo dos valores, se ha utilizado el test exacto de Fisher, por ser más apropiado.

5 Decimos que en una noticia la iniciativa es de la fuente cuando alguna de las fuentes ha tomado la iniciativa. En los casos en que ninguna la ha tomado y sí lo ha hecho algún periodista, se considera que la iniciativa es del periodista. Las informaciones en las que figuran fuentes con iniciativa tanto del periodista como de la propia fuente son calificadas como iniciativa de la fuente. 
Entre los distintos periódicos, se ha detectado que el comportamiento de la iniciativa es significativamente diferente según la temática solo en tres casos: en La Voz de Galicia la iniciativa del periodista se da en el $27,3 \%$ de las noticias institucionales públicas frente al $72,2 \%$ de los casos si el tema no es institucional; en Norte de Castilla la iniciativa corresponde al redactor en el 33,3\% de las informaciones institucionales públicas, pero se produce en el $75 \%$ de las de tema no institucional; y en el Diario de Sevilla el periodista toma la iniciativa en el $38,5 \%$ de las noticias institucionales públicas mientras que lo hace en el $72,7 \%$ cuando la temática es otra.

\subsection{Si el periodista es quien toma la iniciativa independientemente de la temática}

De las 501 noticias que constituyen la muestra del estudio solo 403 son claramente atribuibles, esto es, en el $80,4 \%$ de los casos hay alguna fuente ávida (iniciativa de la fuente) o buscada (iniciativa del periodista). De estas 403, el periodista ha tomado la iniciativa en el 59,6\% de las ocasiones y, por tanto, hay alguna fuente ávida en el $40,4 \%$ de los textos. A este respecto, se detectan claramente diferencias entre los diarios (test chi-cuadrado con $\mathrm{p}$-valor $=0.002$ ). Solo en dos de los diarios analizados hay mayor porcentaje de fuentes ávidas: Ideal, en un $62,5 \%$ de las noticias, y La Voz de Galicia, en un 52,5\%. En el resto, predomina la iniciativa del periodista o del medio, con el Heraldo de Aragón (72,3\%) y El Correo (70,4\%) como los periódicos donde hay mayor número de noticias con fuentes buscadas.

\subsection{Si accede a las fuentes necesarias en noticias que plantean intereses contra- puestos}

Se han encontrado 292 noticias con intereses contrapuestos, un 58,28\% del total de la muestra. De estas 292 informaciones, solo en el 15,4\% de los casos (45) se ha accedido a alguna fuente perjudicada. Si comparamos en este sentido el comportamiento de los diferentes diarios, no se encuentran grandes diferencias (test chi-cuadrado con p-valor $=0.181$ ).

\subsection{Si el número de fuentes suele estar en relación con la calidad de la noticia}

Otro factor clave para medir la calidad del trabajo periodístico es conocer el número de fuentes utilizadas por el redactor en cada noticia. Para ello, este estudio no se considerará al propio periodista como fuente. Así, de las 501 noticias analizadas, el número medio de fuentes detectadas ha sido de casi dos. No obstante, el $48.3 \%$ de las informaciones vienen sustentadas por más de una, mientras que el $8,6 \%$ de las noticias carecen de ellas.

El número de fuentes predominante es una sola (43,1\%), se usan dos en un $24,4 \%$ de las noticias, tres en un $14,2 \%$ y cuatro en un $9,4 \%$ de los casos. Se han localizado hasta un máximo de 5 fuentes por información, si bien esta circunstancia solo se produce en dos ocasiones.

En casi todos los periódicos son más frecuentes las informaciones apoyadas en una única fuente, excepto en Las Provincias, donde predominan por escaso margen las noticias con dos. 
Así queda reflejado en la siguiente tabla

Tabla 1. Fuente: elaboración propia

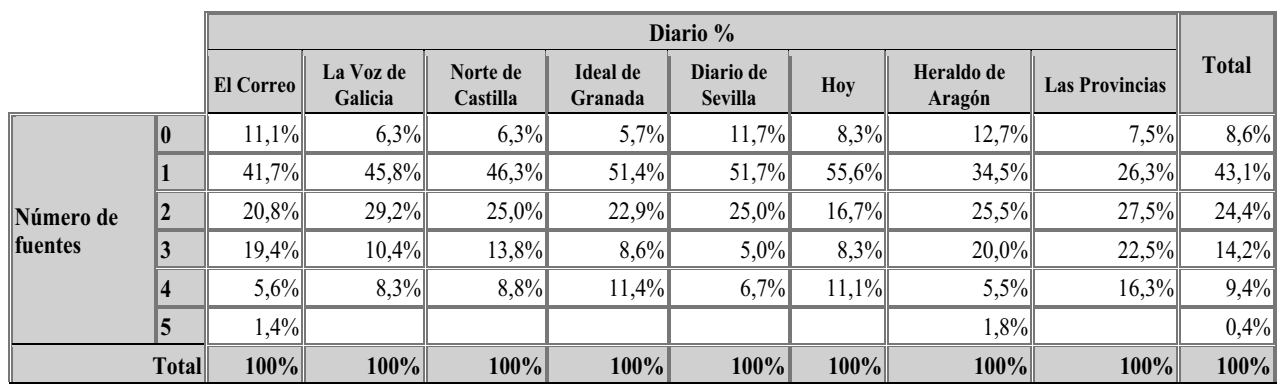

En la agrupación recogida en la Tabla 1 es en la que se han observado mayores diferencias y la que permite afirmar que el número de fuentes utilizado depende del diario al que pertenece la noticia (test chi-cuadrado con $p$-valor=0.017):

Tabla 2. Fuente: elaboración propia

\begin{tabular}{|c|c|c|c|c|c|c|c|c|c|c|}
\hline & & \multicolumn{8}{|c|}{ Diario \% } & \multirow[b]{2}{*}{ Total } \\
\hline & & El Correo & $\begin{array}{l}\text { La Voz de } \\
\text { Galicia }\end{array}$ & $\begin{array}{l}\text { Norte de } \\
\text { Castilla }\end{array}$ & \begin{tabular}{l|} 
Ideal de \\
Granada
\end{tabular} & $\begin{array}{c}\text { Diario de } \\
\text { Sevilla }\end{array}$ & Hoy & $\begin{array}{l}\text { Heraldo de } \\
\text { Aragón }\end{array}$ & $\begin{array}{c}\text { Las } \\
\text { Provincias }\end{array}$ & \\
\hline \multirow{2}{*}{$\begin{array}{l}\text { Número de } \\
\text { fuentes } \\
\text { (agrupado) }\end{array}$} & Menos de 2 & $52,8 \%$ & $52,1 \%$ & $52,5 \%$ & $57,1 \%$ & $63,3 \%$ & $63,9 \%$ & $47,3 \%$ & $33,8 \%$ & $51,7 \%$ \\
\hline & 20 más & $47,2 \%$ & $47,9 \%$ & $47,5 \%$ & $42,9 \%$ & $36,7 \%$ & $36,1 \%$ & $52,7 \%$ & $66,3 \%$ & $48,3 \%$ \\
\hline & Total & $100 \%$ & $100 \%$ & $100 \%$ & $100 \%$ & $100 \%$ & $100 \%$ & $100 \%$ & $100 \%$ & $100 \%$ \\
\hline
\end{tabular}

En casi todos los diarios vemos que más de la mitad de las noticias tienen menos de dos fuentes, a excepción de Las Provincias y Heraldo. La diferencia más llamativa en este sentido se encuentra entre Las Provincias, cabecera en la que el 66,3\% de las noticias poseen al menos dos fuentes, y Hoy y Diario Sevilla, donde este porcentaje alcanza solo el 36,1 y el $36,7 \%$, respectivamente.

\subsection{Número de fuentes según la temática institucional o no institucional}

De las 501 noticias analizadas, 224 (un 44,7\%) tratan de un tema institucional público frente a las 277 (55,3\%) que versan sobre temas de otro ámbito. Para estudiar si existe alguna relación entre el tema de la noticia y el número de fuentes que se han empleado, es preciso ver los porcentajes de informaciones entre 0 y 5 fuentes en cada caso.

6 Tanto en este apartado como en los dos siguientes (2.6 y 2.7), para poder efectuar un análisis más completo del número de fuentes es conveniente realizar algunas agrupaciones de esta variable, de forma que podamos percibir mejor las diferencias existentes entre los distintos diarios, si es que las hubiere. Debido a la gran extensión de las tablas resultantes y a la gran cantidad de agrupaciones que se han probado, mostramos aquellas que puedan ayudar a interpretar de la mejor forma posible las diferencias. 
No parece que exista una gran relación entre el número de fuentes utilizadas y la temática de la información (test chi-cuadrado, p-valor=0.319). Por ello, al igual que en la tabla anterior, ha sido preciso efectuar distintas agrupaciones para buscar aquella que nos permita hallar las mayores diferencias. Así, a partir de la siguiente agrupación (menos de 2 fuentes o al menos 2), ha sido posible detectar cierta relación entre el número de fuentes y el tema de la noticia (nivel de significación 0.01 a través de test exacto de Fisher, p-valor=0.088):

Tabla 3. Fuente: elaboración propia

\begin{tabular}{|c|c|c|c|c|}
\hline & & \multicolumn{2}{|c|}{$\begin{array}{l}\text { Número de fuentes } \\
\text { (agrupado) }\end{array}$} & \multirow[t]{2}{*}{ Total } \\
\hline & & Menos de 2 & 2 o más & \\
\hline \multirow{2}{*}{ Tema \% } & Institucional público & $47,3 \%$ & $52,7 \%$ & $100 \%$ \\
\hline & No institucional público & $55,2 \%$ & $44,8 \%$ & $100 \%$ \\
\hline \multicolumn{2}{|r|}{ Total } & $51,7 \%$ & $48,3 \%$ & $100 \%$ \\
\hline
\end{tabular}

\subsection{Número de fuentes y presencia del periodista}

Un aspecto central de este estudio es ver la relación entre el número de fuentes y la presencia del periodista, ya sea en el lugar donde se produce la noticia (periodismo de calle), por convocatorias o desde la redacción (periodismo de mesa).

Descartando cinco informaciones donde no queda clara cuál es la presencia del periodista, el análisis de las 496 noticias restantes arroja, como datos más destacados, que en los tres supuestos el número de fuentes empleado mayoritariamente ha sido el de una, siendo en las informaciones por convocatorias donde este porcentaje es mayor (49,7\% frente al 40,7\% en el periodismo de mesa y 38,5\% en el de calle). Además, el periodismo de mesa es la tipología donde la proporción de noticias sin fuentes es más amplia ( $12,3 \%$ por $8,4 \%$ en periodismo de calle y $5,2 \%$ en convocatorias), mientras que, en el lado opuesto, el periodismo de calle apoya el 17,3\% de sus informaciones hasta en tres fuentes, un porcentaje que supera a los de periodismo de mesa $(14,8 \%)$ y convocatorias $(10,3 \%)$.

También en este caso ha sido necesario efectuar agrupaciones para clarificar si existen comportamientos distintos. Así, comprobamos que la relación entre la presencia del periodista y el número de fuentes depende del diario (test chi-cuadrado con p-valor $=0.003)$. Entre las mayores diferencias, destaca que mientras el 5,6\% de las noticias de El Correo corresponden a periodismo de calle y disponen de menos de dos fuentes, en Hoy este porcentaje asciende al 30,6\%. Asimismo, mientras que el 5\% las noticias del Diario de Sevilla corresponden a periodismo de calle con dos fuentes, este porcentaje en el Heraldo alcanza el 32,7\%.

Si estudiamos la relación entre número de fuentes y presencia para cada diario de forma separada, aplicando el test chi-cuadrado, en general, y la corrección de Yates para el diario Hoy, se llega a la conclusión de que el único periódico en el que se podría afirmar que existe un comportamiento diferente sería en El Correo, si utilizamos un nivel de significación 0.1 (p-valor $=0.052$ ). En el 73,3\% del periodismo de calle en este diario se usan dos o más fuentes, mientras que en convocatorias y periodismo de mesa las noticias con menos de dos fuentes rondan el $60 \%$. 
A pesar de los resultados obtenidos, cabe precisar que el hecho de que haya un mayor número de fuentes detectables en el periodismo de calle no siempre ha de significar que realmente se usen menos fuentes en el periodismo de mesa, ya que desde la redacción los periodistas suelen hacer uso de un servicio de documentación y tener un acceso directo a otras fuentes propias derivado de su conocimiento y experiencia que muchas veces no son apreciables en el texto.

\subsection{Muchos acontecimientos no se entienden si no se conocen los antecedentes}

En cuanto al grado de contextualización de las noticias a través de la inclusión de los antecedentes, se aprecia que las noticias están mayoritariamente contextualizadas (en un $86 \%$ ).

Al distinguir por diarios, podemos observar que el tratamiento de los antecedentes es bastante dispar: del 94,4\% de información contextualizada en El Correo al $62,5 \%$ en La Voz de Galicia.

Se trata de diferencias significativas a nivel estadístico, ya que el test chi-cuadrado de independencia respecto al diario arroja un p-valor muy próximo a 0 , es decir, el tratamiento de la contextualización depende significativamente del diario elegido.

\subsection{Las consecuencias que se derivan de los acontecimientos deben ser también conocidas por la audiencia}

También la inclusión de las consecuencias que pueden ocasionar las noticias es un elemento clave para contextualizar correctamente las informaciones, si bien hay que tener en cuenta que esta circunstancia dependerá directamente de la tipología y el recorrido de cada noticia.

Desde un punto de vista estadístico, en los diarios analizados el tratamiento de las noticias con consecuencias $(54,5 \%$ de los casos) supera no con mucha diferencia a los textos que no las incluyen $(45,5 \%)$.

Si distinguimos por diarios, obtenemos estos datos:

Tabla 4. Fuente: elaboración propia

\begin{tabular}{|c|c|c|c|c|c|c|c|c|c|}
\hline & \multirow{2}{*}{\multicolumn{8}{|c|}{ Diario \% }} & \multirow[b]{3}{*}{ Total } \\
\hline & & & & & & & & & \\
\hline & El Correo & $\begin{array}{l}\text { La Voz de } \\
\text { Galicia }\end{array}$ & $\begin{array}{l}\text { Norte de } \\
\text { Castilla }\end{array}$ & $\begin{array}{l}\text { Ideal de } \\
\text { Granada }\end{array}$ & $\begin{array}{l}\text { Diario de } \\
\text { Sevilla }\end{array}$ & Hoy & $\begin{array}{l}\text { Heraldo de } \\
\text { Aragón }\end{array}$ & $\begin{array}{c}\text { Las } \\
\text { Provincias }\end{array}$ & \\
\hline $\begin{array}{l}\text { Información con } \\
\text { consecuencias }\end{array}$ & $52,8 \%$ & $39,6 \%$ & $40,0 \%$ & $68,6 \%$ & $43,3 \%$ & $61,1 \%$ & $47,3 \%$ & $77,5 \%$ & $54,5 \%$ \\
\hline $\begin{array}{l}\text { Información sin } \\
\text { consecuencias }\end{array}$ & $47,2 \%$ & $60,4 \%$ & $60,0 \%$ & $31,4 \%$ & $56,7 \%$ & $38,9 \%$ & $52,7 \%$ & $22,5 \%$ & $45,5 \%$ \\
\hline Total & $100 \%$ & $100 \%$ & $100 \%$ & $100 \%$ & $100 \%$ & $100 \%$ & $100 \%$ & $100 \%$ & $100 \%$ \\
\hline
\end{tabular}

A simple vista, se puede apreciar que el tratamiento de las consecuencias es mayor en diarios como Las Provincias (77,5\% de las noticias) o Ideal (68,6\%), mientras que La Voz solo alcanza el 39,6\%. En el plano estadístico son diferencias significativas, pues el p-valor del test chi-cuadrado de independencia da un valor muy próximo a cero. Por tanto, existe una dependencia significativa del tratamiento de las noticias respecto al diario elegido. 


\subsection{La identificación de las fuentes es otro de los parámetros de calidad}

Este estudio se efectúa desde dos puntos de vista: analizando directamente todas las fuentes detectadas en las noticias (excluyendo el propio periodista como fuente) o bien analizando las noticias y estudiando en ellas la presencia de fuentes anónimas o no atribuidas.

\subsubsection{Desde el punto de vista de las fuentes}

En las 501 noticias analizadas, se han localizado 871 fuentes. De ellas, el 81,3\% están identificadas, el 15\% están atribuidas y el 3,7\% son anónimas.

Al hacer un estudio ${ }^{7}$ de los diarios por separado, vemos que las fuentes identificadas superan el $75 \%$ en todos los casos, destacando Hoy con un $93 \%$ de ellas. Respecto a las fuentes atribuidas de un modo indefinido ("fuentes próximas a la investigación"), la mayor parte de los rotativos tienen entre un $17,3 \%$ y un $20,6 \%$. El diario con mayor proporción de fuentes no identificadas es el Diario de Sevilla $(5,8 \%)$.

Tabla 5. Fuente: elaboración propia

\begin{tabular}{|c|c|c|c|c|c|c|c|c|c|c|}
\hline & & \multirow{2}{*}{\multicolumn{8}{|c|}{ Diario \% }} & \multirow[b]{3}{*}{ Total } \\
\hline & & & & & & & & & & \\
\hline & & El Correo & $\begin{array}{c}\text { La Voz de } \\
\text { Galicia }\end{array}$ & $\begin{array}{c}\text { Norte de } \\
\text { Castilla }\end{array}$ & \begin{tabular}{l|l} 
Ideal de \\
Granada
\end{tabular} & $\begin{array}{l}\text { Diario de } \\
\text { Sevilla }\end{array}$ & Hoy & $\begin{array}{l}\text { Heraldo de } \\
\text { Aragón }\end{array}$ & $\begin{array}{c}\text { Las } \\
\text { Provincias }\end{array}$ & \\
\hline \multirow{3}{*}{ Atribución } & Identificada & $85,4 \%$ & $80,2 \%$ & $87,7 \%$ & $78,8 \%$ & $75,6 \%$ & $93,0 \%$ & $77,3 \%$ & $76,6 \%$ & $81,3 \%$ \\
\hline & Atribuida & $9,8 \%$ & $17,3 \%$ & $8,7 \%$ & $18,6 \%$ & $18,6 \%$ & $5,3 \%$ & $20,6 \%$ & $18,7 \%$ & $15,0 \%$ \\
\hline & Anónima & $4,9 \%$ & $2,5 \%$ & $3,6 \%$ & $2,5 \%$ & $5,8 \%$ & $1,8 \%$ & $2,1 \%$ & $4,7 \%$ & $3,7 \%$ \\
\hline \multicolumn{2}{|r|}{ Total } & $100 \%$ & $100 \%$ & $100 \%$ & $100 \%$ & $100 \%$ & $100 \%$ & $100 \%$ & $100 \%$ & $100 \%$ \\
\hline
\end{tabular}

\subsubsection{Desde el punto de vista de las propias noticias}

Al fijarnos en cada una de las noticias analizadas, vemos que solo hay alguna fuente anónima en el 5,8\% de los casos. Al efectuar un estudio comparado entre diarios, los resultados muestran que no existe en este sentido una diferencia significativa entre unos y otros (test chi-cuadrado con corrección de Yates, p-valor próximo a 1). Así, vemos cómo el porcentaje de informaciones con alguna fuente anónima oscila entre el 3,6\% de los casos en Heraldo, 4,3\% de Ideal, 5,6\% de Hoy, 6,3\% de Norte de Castilla, Las Provincias y La Voz de Galicia, 6,7\% de Diario de Sevilla y El Correo, con el $6,9 \%$.

7 Para este estudio, al centrarse en las fuentes en vez de en las noticias, no podemos aplicar estadística inferencial (es decir, tests como los aplicados anteriormente, chi-cuadrado, test exacto de Fisher,...), ya que hay distintas fuentes que proceden de la misma noticia, por lo que puede haber cierta relación entre ellas. Por ello, hemos calculado el coeficiente V de Cramer, para sacar conclusiones desde el punto de vista descriptivo. Este coeficiente toma valores entre 0 y 1 . Cuanto más cercano sea el valor a 0 , mayor será la asociación, es decir, la relación o dependencia. Cuanto más cercano sea el coeficiente a 0 , más cercana a la independencia será la situación, es decir, más débil será la asociación o dependencia. El coeficiente V-Cramer con valor 0.113 , indica una asociación o relación débil entre diario y atribución. 


\subsection{Relación entre noticias institucionales y fuentes institucionales}

En las noticias institucionales, hemos observado 415 fuentes (sin incluir al periodista). De ellas, la mayor parte son institucionales $(61,2 \%)$, seguidas de personales o no institucionales $(26 \%)$ y documentales $(12,5 \%)$. Solo un $0,2 \%$ de los casos procede de un experto.

En todos los diarios predominan las fuentes institucionales, seguidas de las personales o no institucionales, salvo en La Voz de Galicia e Ideal, donde la proporción de fuentes documentales se sitúa en segundo lugar.

\section{Conclusiones}

A tenor de los resultados obtenidos en el presente estudio, podemos concluir que en la prensa local o de proximidad el porcentaje de noticias que cuenta con presencia del periodista en el lugar de los hechos supera ligeramente o es similar al periodismo de mesa y al de convocatorias. Se observa una mayor presencia de fuentes ávidas en el ámbito institucional público que en otras temáticas, donde predomina la iniciativa del periodista o del medio, y solo a veces los periódicos acceden a las fuentes necesarias cuando las noticias tienen intereses contrapuestos.

En casi todos los periódicos son más frecuentes las informaciones apoyadas en una sola fuente que en dos o más, lo que representa una proporción baja como medidor de la calidad del trabajo periodístico, si bien hay que tener en cuenta que la gestión de las fuentes que se pueda efectuar en el periodismo de mesa no siempre es fácilmente detectable en el análisis de los textos.

En el periodismo local, las noticias están mayoritariamente bien contextualizadas al incluir los antecedentes y, en función de la temática, las consecuencias, con lo que se favorece que la audiencia comprenda bien los acontecimientos. Además, las fuentes aparecen identificadas en la gran mayoría de las ocasiones, un hecho que refuerza la credibilidad de los periodistas y los medios ante su público más próximo. Tampoco en este ámbito el uso de las fuentes anónimas suele estar justificado.

Uno de los aspectos más destacables es la poca presencia de los periodistas en el lugar donde se producen las noticias, apenas un tercio, una proporción muy baja si tomamos en consideración que se trata de los temas de mayor relieve en cada página y los que se supone que cada medio debe priorizar más por su singularidad y la mayor accesibilidad a las fuentes. También llama la atención el escaso número de fuentes empleadas por noticia teniendo en cuenta que es en este tipo de periodismo donde se precisa de un mayor número de ellas al compartir un mismo espacio periodistas, fuentes y receptores de la información, y que suelen ser las informaciones más elaboradas en un diario.

\section{Referencias bibliográficas}

ALESSANDRI, Francisca et al. (2001): "VAP: un sistema métrico de la calidad periodística", Cuadernos de Información, 14, pp. 112-120.

BEZUNARTEA, Ofa (1988): Noticias e ideología profesional. Los periodistas vascos en la transición. Bilbao, Ediciones Deusto. 
BEZUNARTEA, Ofa et al. (1998): 21 Lecciones de reporterismo. Bilbao, Servicio Editorial UPV-EHU.

CASAS, José M. (1997): Inferencia Estadística. Madrid, Editorial Centro de Estudios Ramón Areces, S.A.

CASERO RIPOLLÉS, Andreu y LÓPEZ RABADÁN, Pablo (2013): "La Gestión de fuentes informativas como criterio de calidad profesional", en GÓMEZ MOMPART, Josep Lluís; GUTIÉRREZ LOZANO, Juan Francisco; PALAU SAMPIO, Dolors (eds., 2013): La Calidad Periodística. Teorias, Investigaciones y Sugerencias Profesionales. Col. lecció Aldea Global. Bellaterra, Universitat Autònoma de Barcelona; Castelló de la Plana, Uni-versitat Jaume I; Barcelona, Universitat Pompeu Fabra; València, Universitat de València, pp. 73-90.

DIEZHANDINO, Pilar (coord.) (2007): Periodismo en la era de internet. Barcelona, Ariel.

FISHMAN, Mark (1980): La fabricación de la noticia. Buenos Aires, Ediciones Tres Tiempos.

FONTCUBERTA, Mar (1999): "Pauta y calidad informativa", Cuadernos de Información, $\mathrm{n}^{\mathrm{o}}$ 13, pp.61-69.

GALLEGO DÍAZ, Soledad (2013): "Perros Guardianes. Periodismo de investigación y su contribución a la democracia". Periodistas. Revista de la FAPE, n 32.

GANS, Herbert J. (1980): Deciding What's News: a study of CBS evening news, NBC nightly news, Newsweek and Time. London, Constable.

GARCÍA GORDILLO, María del Mar; BEZUNARTEA VALENCIA, Ofa; y RODRÍGUEZ CRUZ, Inés (2013): "El Valor Agregado Periodístico, herramienta para el periodismo de calidad", en GÓMEZ MOMPART, Josep Lluís.; GUTIÉRREZ LOZANO, Juan Francisco; PALAU SAMPIO, Dolors (eds., 2013). La Calidad Periodística. Teorías, Investigaciones y Sugerencias Profesionales. Col·lecció Aldea Global. Bellaterra, Universitat Autònoma de Barcelona; Castelló de la Plana, Universitat Jaume I; Barcelona, Universitat Pompeu Fabra; València, Universitat de València, pp. 39-52.

GIEBER, Walter (1964): "News Is What Newspaper Men Make It", en: DEXTER, Lewis, A. and WHITE, David M. (Eds): People Society and Mass Communication. London, The Free Press of Glencoe, pp. 289-297.

HEIDER, Don; MCCOMBS, Maxwell; and POINDEXTER, Paula M. (2005): "What the Public Expects of Local News: Views on Public and Traditional Journalism". Journalism \& Mass Communication Quarterly. 82: 952-967 (Winter 2005)

KOVACH, Bill and ROSENSTIEL, Tom (2003): Los elementos del periodismo. Madrid, Santillana Ediciones.

LIPPMANN, Walter (1965): Public Opinion. New York, Free Press.

O'NEILL, Deindre; O'CONNOR, Catherine (2008): “The Passive Journalist". Journalism Practice 2: 3, 498. 
PELLEGRINI, Silvia. et al. (2011): Valor agregado periodístico. La apuesta por la calidad de las noticias. Santiago, Ediciones Universidad Católica de Chile.

SCHUDSON, Michael (2003): The Sociology of News. New York, Norton.

SIGAL, Leon V. (1973): Reporters and Officials: the organisation and politics of newsmaking. Lexington, MA, Lexington Books.

SIGAL, Leon V. (1986): "Sources Make the News", en: MANOFF, Robert Karl and SCHUDSON, Michael (Eds.): Reading the News. New York, Pantheon Books, p. 9.

STRENTZ, Herbert (1989): News Reporters and News Sources. Ames, IA, Iowa State University Press.

TÉRAMO, María Teresa (2006): “Calidad de la información periodística en Argentina. Estudio de diarios y noticieros". Palabra-Clave, vol. 9, 1, pp. 57-84.

TUCHMAN, Gaye (1978): Making News. A Study in the Construction of reality. New York, Free Press. 